\title{
Improving standards in flexible bronchoscopy for lung cancer
}

\author{
M.G. Slade*, N.M. Rahman", A.E. Stanton`, L. Curry ${ }^{+}$, G.C. Slade*, \\ C.A. Clelland ${ }^{\S}$ and F.V. Gleeson ${ }^{f}$
}

ABSTRACT: Can the detection rate of flexible bronchoscopy for lung cancer be increased by a series of simple quality improvement measures?

Bronchoscopy-associated clinical parameters were prospectively recorded between 2001 and 2007 in patients with suspected lung malignancy. The detection rate of bronchoscopy, diagnostic yield of each biopsy modality and the possible impact of different service-improvement measures were assessed.

746 bronchoscopies were performed in 704 patients. The detection rate of bronchoscopy for malignancy was $83.6 \%$, and increased over time (67.3\% detection rate in 2001 (95\% Cl 52.9-79.7), $89.7 \%$ detection rate in 2007 (95\% Cl 81.3-95.2); $p<0.001)$. Detection rate increased for bronchoscopically visible $(75.0 \%$ in 2001 to $94.5 \%$ in 2007$)$ and non-visible tumours $(41.7 \%$ in 2001 to $81.2 \%$ in $2007 ; p<0.001$ for both analyses). Prior computed tomography availability was associated with a higher diagnostic yield that did not reach statistical significance. Logistic regression analysis identified tumour visibility, year of study, use of transbronchial needle aspiration and pathologist identity as independent predictors of a positive diagnosis.

A significant increase in bronchoscopic detection rate for malignancy occurred in association with a number of simple improvement measures.

KEYWORDS: Bronchoscopy, carcinoma nonsmall cell lung, lung neoplasms, quality-assurance healthcare, small cell lung carcinoma

ung cancer is the leading cause of death from malignant disease in the developed world, causing $22 \%$ of cancer deaths in the UK in 2007 [1]. Rapid, accurate pathological diagnosis and staging are vital for good patient care. Flexible bronchoscopy (FB) is perhaps the most important single technique in lung cancer diagnosis and, therefore, maximising the detection rate of FB should be a key objective.

The detection rate (or diagnostic sensitivity) of FB for malignancy varies widely in published studies [2]. It is higher when the endobronchial tumour is visible than when peripheral nodules not visible endobronchially are sampled. Prior computed tomography (CT) scanning increases the detection rate of FB for malignancy [3] and is recommended in national guidelines [4]. Within our study where CT scans are referred to, they are taken to be contrast-enhanced scans of the chest and upper abdomen. Transbronchial needle aspiration (TBNA) $[5,6]$ and more sophisticated techniques, such as endobronchial ultrasound (EBUS) $[7,8]$ and electromagnetic navigation bronchoscopy $[9,10]$, can further increase the detection rate. However, the vast majority of lung cancer bronchoscopy in the UK is performed by practitioners who lack access to, or training in, these modalities.

From 2001, a series of measures aimed at quality improvement in bronchoscopy were introduced and a continuous, prospective audit of detection rate was started.

\section{METHODS}

Consecutive patients undergoing bronchoscopy for suspected lung malignancy from July 2001 to December 2007 were entered prospectively into the audit. The chair of the Oxford Ethics Committee (John Radcliffe Hospital, Oxford, UK) confirmed that an application for approval was not required for this study, which assesses the quality of routine patient care.

\section{AFFILIATIONS}

*Dept of Thoracic Oncology,

Papworth Hospital NHS Foundation

Trust, Papworth Everard,

${ }^{\#}$ Oxford Centre for Respiratory

Medicine, Churchill Hospital,

${ }^{f}$ Dept of Radiology, Churchill

Hospital, and

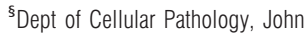

Radcliffe Hospital, Oxford,

"Dept of Respiratory Medicine, Great

Western Hospital, Swindon, and

${ }^{+}$Dept of Respiratory Medicine,

Wycombe Hospital, High Wycombe, UK.

\section{CORRESPONDENCE}

M.G. Slade

Dept of Thoracic Oncology

Papworth Hospital NHS Foundation

Trust

Papworth Everard

Cambridge

CB23 3RE

UK

E-mail: Mark.slade@nhs.net

Received:

June 232010

Accepted:

July 132010

First published online:

Aug 062010 


\section{Inclusion criteria}

1) Patients undergoing bronchoscopy from July 2001 to December 2007, and 2) suspected lung malignancy detected from chest radiograph (CXR) or CT.

\section{Exclusion criteria}

1) Normal CXR prior to procedure, and 2) lung malignancy not suspected prior to bronchoscopy.

\section{Data collected}

Along with demographic data, data recorded were: 1) whether $\mathrm{CT}$ was present for the procedure; 2) visual classification of tumour (see below); 3) diagnostic modalities employed (i.e. bronchial biopsy, brushing or washing, or TBNA); 4) identity of bronchoscopist and reporting pathologist; 5) results of each diagnostic sample; 6) whether FB was diagnostic of malignancy; and 7) final diagnosis.

Visual classification of endobronchial appearance was divided into one of three categories: definite endobronchial tumour (e.g. exophytic mass within the bronchus); possible tumour (oedema, erythema constriction or compression); or no visible abnormality. The operator entered data prospectively at the time of bronchoscopy. The histocytopathological result of each sample was classified as follows: definite malignancy was considered positive and all other results, including "highly suspicious of malignancy" and similar descriptions were considered negative. In patients with negative FB, a final diagnosis was confirmed by: alternative histocytological sampling, establishment of a benign disease or follow-up until death.

\section{Bronchoscopy}

All bronchoscopy procedures were performed or supervised by consultant respiratory physicians. A video bronchoscopy system was used (EB1830T3 bronchoscopes and an EPM1000 processor; Pentax Medical, Slough, UK). The selection and sequence of sampling techniques were left to the operator. For visible endobronchial tumour, forceps biopsy, brushing and washing samples were obtained prior to January 2003. After this date, bronchial washings were no longer routinely performed in the presence of probable or definite tumour [11] following an audit demonstrating no significant additive diagnostic yield. TBNA was used to sample accessible lymph nodes, submucosal abnormalities or necrotic endobronchial lesions. Fluoroscopy and rapid onsite cytological examination were not used.

During the period 2001-2007, a number of changes were introduced to the bronchoscopy service. These included: 1) in 2001 the appointment of a lead clinician and lead nurse for bronchoscopy; 2) increasing emphasis on obtaining a CT scan prior to $\mathrm{FB}$, permitting increased clinicoradiological collaboration prior to $\mathrm{FB}$; 3) increasing use of distal bronchial brushings to sample tumours not visible endobronchially, using the method described by LeE et al. [12]; 4) in March 2002 bronchial brush diameter was changed from $2 \mathrm{~mm}$ (Olympus BC202D-2010; Olympus Keymed, Southend-on-Sea, UK) to $5 \mathrm{~mm}$ diameter (Olympus BC202D-5010; Olympus Keymed); and 5) routine use of TBNA by the lead clinician for bronchoscopy from August 2002.
These changes were intended as quality improvement measures. In addition, a change occurred in the reporting pathologists. Pathologist A was reporting until retirement in March 2002. Pathologist B and C were reporting from July 2001 until the end of the audit period.

\section{Outcome measures}

The primary outcome measure was the change over time in the detection rate of bronchoscopy in the diagnosis of lung malignancy. A diagnosis of lung malignancy was considered secure when histologically confirmed by any method, or when clinical follow-up was consistent with lung malignancy in the absence of a histocytological diagnosis.

Secondary outcome measures were the impact of: CT availability, TBNA and the type of bronchial brushes used, upon the detection rate of bronchoscopy for lung malignancy. The detection rate of individual biopsy techniques, and of FB overall, was studied using summary statistics and statistical modelling (logistic regression) to examine predictive factors for a positive diagnosis, including time.

Tests for trend in proportion were conducted using the Cochran-Armitage trend test (STATA version 9; Stata Corp., College Station, TX, USA). Measures of association (Chisquared analysis) and logistic regression models were also used (SPSS version 14; SPSS Inc., Chicago, IL, USA).

Details of protocols for CT scanning and pathology specimen preparation are available in an online supplement.

\section{RESULTS}

\section{Summary data}

Between July 2001 and December 2007, 746 bronchoscopies were performed on 704 patients. Patients age ranged from 29 yrs to 94 yrs (mean \pm SD $69.1 \pm 10.6$ yrs). The majority of patients were male $(n=446,63.4 \%)$. A final malignant diagnosis was made in 631 (89.6\%) out of 704 patients referred with suspected intrathoracic malignancy. In 553 out of 631 patients (87.6\% true positive, 95\% CI 85.1-90.2\%) this diagnosis was confirmed bronchoscopically. In 11 patients there was radiological progression consistent with lung malignancy without histological confirmation. Of the 73 patients with a benign diagnosis this was based on histological findings in four patients (organising pneumonia, hamartoma, tuberculosis and sarcoidosis) and on sequential radiological examination showing stability or resolution in the remaining 69 patients. A detailed breakdown of final diagnoses is shown in table 1.

Overall numbers of diagnostic FB for lung malignancy decreased over the study period; 114 in the first 12-month period versus 95 in the last 12-month period. The total number of FBs conducted and their indication is shown in table 2.

\section{Change in detection rate}

There was a statistically significant improvement in overall detection rate of FB for lung malignancy from $67.3 \%$ (95\% CI $52.9-79.7)$ in 2001 to $89.7 \%$ (95\% CI 81.3-95.2) in 2007 (Cochran-Armitage test for trend $p=0.003$ ) (table 3). The detection rate when the tumour was visible endobronchially increased from $75.0 \%$ (95\% CI 58.8-87.3) in 2001 to $94.5 \%$ (95\% CI 84.9-98.9) in $2007(\mathrm{p}=0.006)$, and when the tumour was not visible from $41.7 \%$ (95\% CI 15.2 to 72.3 ) in 2001 to $81.2 \%$ 
TABLE 1 Final diagnosis in all patients undergoing flexible bronchoscopy for suspected lung malignancy

\begin{tabular}{|c|c|c|}
\hline Diagnosis & Total group & Bronchoscopic diagnosis of malignancy \\
\hline \multicolumn{3}{|c|}{ Nonsmall cell lung cancer } \\
\hline Squamous cell & $228(157: 71)$ & 223 \\
\hline Adenocarcinoma & $120(67: 53)$ & 106 \\
\hline Unclassified & $143(93: 50)$ & 111 \\
\hline Carcinoid & 12 & 12 \\
\hline \multicolumn{3}{|l|}{ Intrathoracic metastases } \\
\hline Breast & 5 & 3 \\
\hline Colorectal & 5 & 4 \\
\hline Renal cell & 5 & 4 \\
\hline Oesophageal & 4 & 2 \\
\hline Haemangioblastoma & 1 & \\
\hline Lymphoma & 5 & 2 \\
\hline Carcinosarcoma & 2 & 2 \\
\hline Presumed lung cancer & 11 & \\
\hline Benign disease & 73 & \\
\hline Total & 704 & 553 \\
\hline
\end{tabular}

(95\% CI 63.6 to 92.8$)$ in $2007(\mathrm{p}=0.005)$. Overall sensitivities over time (30 procedure per interval) are demonstrated in figure 1 for cases overall (fig. 1a) and for visible versus nonvisible tumours (fig. 1b). All biopsy modalities increased in detection rate over time (table 3, fig. 2), with the exception of brush biopsies in visible tumours.

\section{Impact of CT}

The availability of CT increased over the study period (table 3). For both bronchoscopically visible and non-visible tumours CT prior to the procedure was associated with an increased detection rate, but this did not reach statistical significance. Visible tumour: no CT performed, detection rate 177 (83.2\%) out of 203; CT performed, detection rate $243(92.0 \%)$ out of 264 ; Chi-squared 1 degree of freedom $(\mathrm{df})=2.99, \mathrm{p}=0.08$. Nonvisible tumour: no CT performed, detection rate $33(56.9 \%)$ out of 58; CT performed, detection rate $97(70.3 \%)$ out of 138; Chisquared $1 \mathrm{df}=3.28, \mathrm{p}=0.07$.

\section{Impact of service provision changes on detection rate}

A logistic regression model was used to assess the overall impact of measured factors on the likelihood of a positive bronchoscopic diagnosis of lung malignancy. The year of study was initially assessed to account for measured and unmeasured variable change over time. Subsequent variables were assessed individually with year of study and, if significant,

TABLE 2 Number of flexible bronchoscopies (FBs) performed per year

\begin{tabular}{|c|c|c|c|c|c|c|c|c|}
\hline & $2001^{\#}$ & 2002 & 2003 & 2004 & 2005 & 2006 & 2007 & Total \\
\hline Total FBs & 59 & 113 & 148 & 125 & 97 & 109 & 95 & 746 \\
\hline FBs in patients with final diagnosis of malignancy & 52 & 106 & 138 & 104 & 86 & 96 & 87 & 669 \\
\hline FBs positive for malignancy $^{+}$ & 35 & 85 & 117 & 83 & 75 & 82 & 78 & 555 \\
\hline
\end{tabular}

Data are presented as $n$ or \%. ${ }^{*}$ : ran from July to December only; ": total number of FBs in patients with malignancy exceeds the total number of patients diagnosed with malignancy because repeat FB was performed following prior false-negative FB or because of tumour relapse; ${ }^{+}$: the total number of FBs positive for malignancy exceeds the total number of patients diagnosed with malignancy bronchoscopically because two patients underwent repeat bronchoscopy to diagnose tumour relapse. 
TABLE 3 Detection rate of bronchoscopy for lung malignancy in patients with a final diagnosis of malignancy

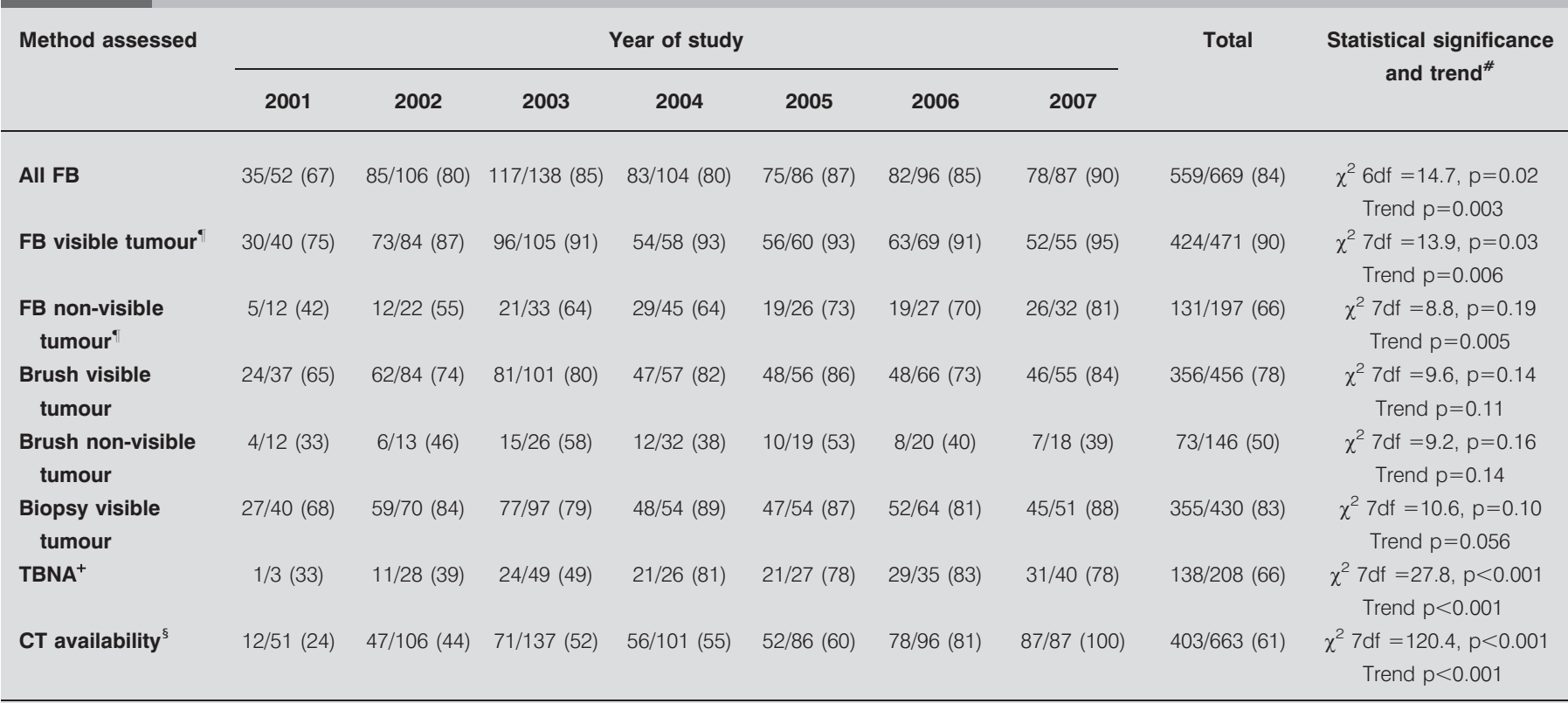

Data are presented as true positive/true positive + false negative (\%), unless otherwise stated. Data for brush and biopsy visible tumour are presented as detection rate for each sampling modality. False positive rate of bronchoscopy for lung cancer was 0\%. FB: flexible bronchoscopy; TBNA: transbronchial needle aspiration; CT; computed tomography; df: degree of freedom. ${ }^{*}$ : Cochran-Armitage trend test; " : in five patients FB tumour visibility was not recorded; ${ }^{+}$: number of TBNA samples positive for malignancy/total number of TBNA samples obtained from patients with a final diagnosis of malignancy; ${ }^{\text {s: }}$ CT availability prior to bronchoscopy in patients with a final diagnosis of lung malignancy only.

were included in the final model. Two separate models were constructed to assess the predictive factors for a positive bronchoscopic diagnosis with all procedures and then with non-visible tumour cases only.

For the all-cases combined model, the identity of the operator and prior CT scan were not significantly associated with a positive bronchoscopic diagnosis. Identity of the reporting pathologist (2df, $\mathrm{p}=0.006)$, visibility of the tumour (1df, $\mathrm{p}<0.001)$ and use of TBNA $(1 \mathrm{df}, \mathrm{p}=0.035)$ were independent predictors of a positive bronchoscopic diagnosis, with year of study $(1 \mathrm{df}, \mathrm{p}=0.09)$ showing a trend towards prediction.

For the non-visible tumour model, identity of pathologist or operator, CT prior to bronchoscopy and use of TBNA were not statistically significantly associated with a positive bronchoscopic diagnosis. Year of study $(1 \mathrm{df}, \mathrm{p}=0.011)$ was the only independent predictor of a positive bronchoscopic diagnosis.

As year of study and availability of CT were highly associated (table 3), an interaction term combining year of study and CT availability was constructed, which showed no significant interaction between the two.

\section{Impact of bronchial brush diameter}

Bronchial brush diameter was changed in April 2002 (after 89 procedures in the audit) from $2 \mathrm{~mm}$ to $5 \mathrm{~mm}$. When visible tumours were sampled, 2-mm brushes were associated with a significantly lower diagnostic yield than 5-mm brushes: 2-mm diagnostic yield was $39(67.2 \%)$ out of 58 and 5-mm diagnostic yield was $298(80.1 \%$ ) out of 372, (Chi-squared $1 \mathrm{df}=4.9$, $\mathrm{p}=0.027)$. Comparing a non-visible tumour in which a diagnostic brush was attempted, 2-mm brushes were again associated with a significantly lower diagnostic yield (2-mm diagnostic yield five $(35.7 \%)$ out of 14 and $5-\mathrm{mm}$ diagnostic yield $61(51.7 \%)$ out of 118 (Chi-squared $1 \mathrm{df}=5.43, \mathrm{p}=0.02)$ ).

\section{DISCUSSION}

There was a significant and continuing improvement in detection rate of FB for thoracic malignancy over the audit period, from $67.3 \%$ to $89.7 \%$. This occurred in association with improvement measures that are either universally adoptable (e.g. pursuit of CT scanning prior to FB and change in bronchial brush size), or require only modest resources to implement (e.g. TBNA). These results, therefore, have more wide-spread relevance than in specialist centres in resource-rich countries alone. The proportional improvement was greatest for nonvisible tumours, where the detection rate improved from $41.7 \%$ to $81.2 \%$ over the study period.

Our audit of 746 FB in 704 patients is one of the larger reported series. There are three comparable published series [13-15]. We believe our study is the first to assess prospectively the change in detection rate over time, with all FB procedures recorded in exact sequential order. FB detection rate for both central (visible) and peripheral bronchogenic carcinoma has recently been systematically reviewed on behalf of the American College of Chest Physicians (ACCP) [2]. For visible tumours, the overall detection rate from 35 studies reporting on a total of 4,507 patients was $88 \%$, while for peripheral tumour, in 34 studies reporting on 5,742 patients, it was 78\%. Current British Thoracic Society (BTS) guidelines [16] suggest a target detection rate for FB of $80 \%$ for visible endobronchial tumour. 

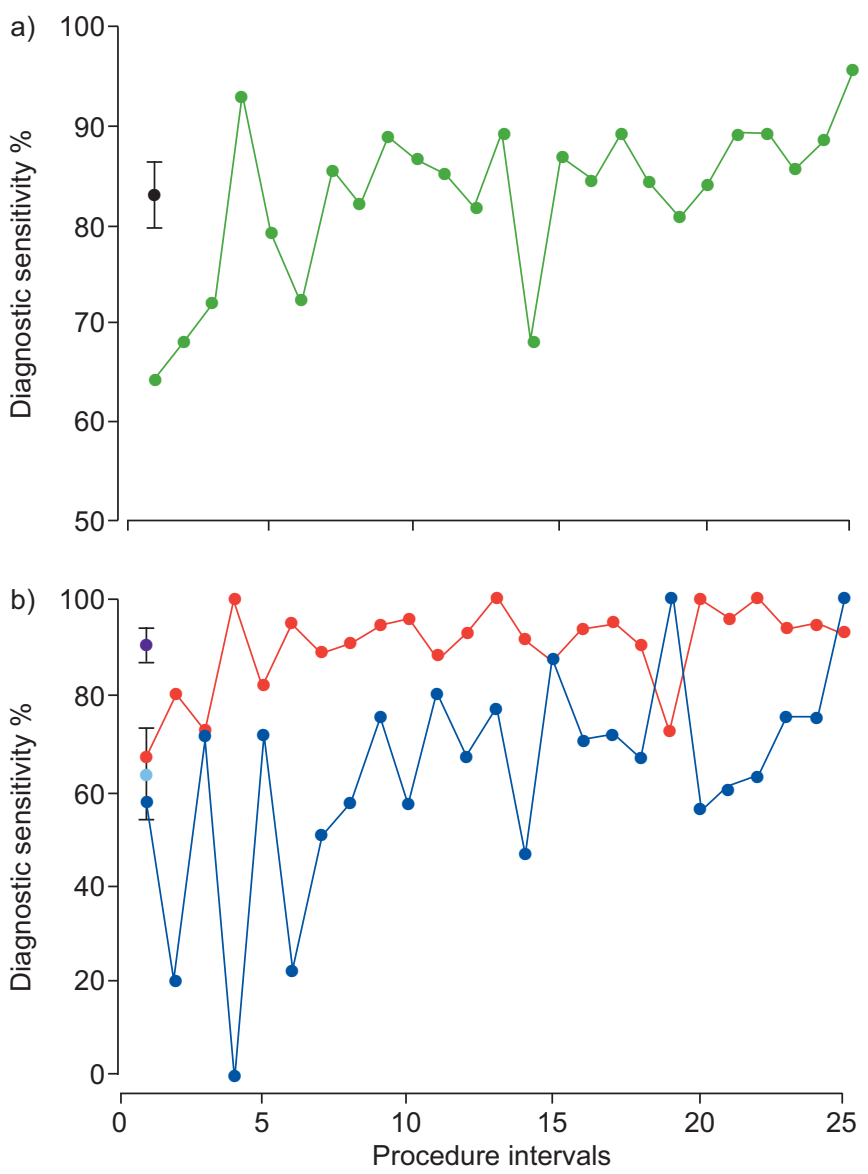

FIGURE 1. Flexible bronchoscopy detection rate over time (30 procedures per interval). a) Overall detection rate and b) detection rate for visible versus non-visible tumours. - overall sensitivity and $95 \% \mathrm{Cl}$; green: overall diagnostic sensitivity; red visible tumour; purple: visible tumour overall sensitivity and $95 \% \mathrm{Cl}$; blue: nonvisible tumour; pale blue: non-visible tumour overall sensitivity and $95 \% \mathrm{Cl}$.

We believe the evidence from our own study and the ACCP review [2] suggests that this target is set unacceptably low.

We believe that our study is the first to demonstrate the impact over time of the introduction of TBNA within a lung cancer service. A learning curve is evident, with the percentage of diagnostic specimens increasing from $50 \%$ in 2003 to $78 \%$ in 2007 (fig. 2). This effect is expected and has been described previously $[17,18]$, although a recent study reported high initial diagnostic yield by a team of experienced bronchoscopists [19]. TBNA requires no capital investment, was first described via FB more than 25 yrs ago, is cost-effective and very safe $[5,20]$. It is, however, under utilised in both UK and US hospitals [21, 22] with only $27 \%$ of UK bronchoscopists reporting having used the technique in the preceding 12 months [22]. We believe that in each hospital where FB is performed for diagnosing lung cancer, there should be at least one operator who develops skills in TBNA. SHAH et al. [6] reported the impact of TBNA use in lung cancer diagnosis and staging in two teaching hospitals in London, UK. They found that TBNA was the sole diagnostic modality in $30(7 \%)$ out of 433 lung cancer patients who underwent FB [6]. TBNA has an established role in the sampling of mediastinal and hilar lymph nodes [23, 24] and submucosal lesions [25], and has

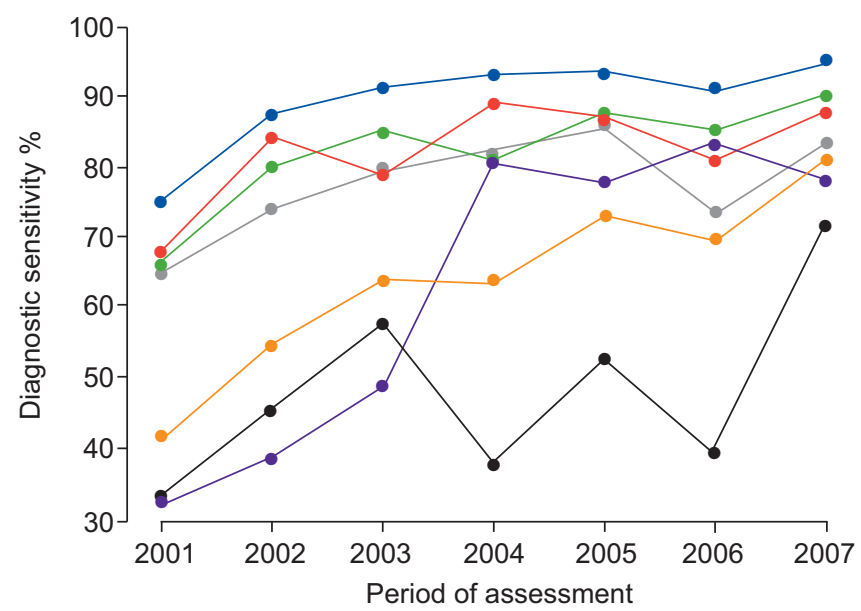

FIGURE 2. Sensitivity of bronchoscopic diagnostic techniques over time. Blue: flexible bronchoscopy (FB) visible tumour; green: overall FB; red: biopsy with visible tumour; grey: brush with visible tumour; orange: FB non-visible tumour; purple: transbronchial needle aspiration; black: brush non-visible tumour.

been proposed for sampling peripheral pulmonary nodules [26] and endobronchial tumour [27]. In our centre (Oxford Centre for Respiratory Medicine, Oxford) it has been used exclusively for sampling mediastinal and hilar lymph nodes and submucosal tumour. EBUS-TBNA has shown increased detection rate for staging mediastinal and hilar lymph nodes in lung cancer compared with conventional TBNA and it is likely that this technique will grow in importance. It does, however, require significant training and capital investment.

Previous studies have found that a pre-procedure CT scan increases FB diagnostic yield. In a randomised two-group study [3], all patients with suspected lung cancer underwent CT scanning. In group A, the results of the CT were reviewed before FB, allowing a change of biopsy procedure if indicated. In group $B$, all patients proceeded to FB with the bronchoscopist blinded to the CT result. Detection rate of FB was $89 \%$ in group A but $71 \%$ in group B $(p=0.012)$. CT scanning can be expected to increase the diagnostic yield of bronchoscopy for peripheral lung malignancy by providing an accurate segmental location for the primary tumour, or an extrabronchial target for TBNA. The presence on a CT scan of a bronchus leading to the lesion is known to increase the detection rate [28]. The failure to demonstrate an independent effect of prior CT scanning on the detection rate for non-visible tumours is particularly surprising in our study, given the difficulty the bronchoscopist encounters in selecting a target for sampling when no CT is available and no abnormality is visible endobronchially. The absence of a demonstrated effect may be due to generally increased CT scanning prior to bronchoscopy which occurred over the course of the study, in association with a number of measured and non-measured variables resulting in increased diagnostic yields in later years, masking the added value of CT scanning. Lest it be thought that the need for CT prior to lung cancer bronchoscopy is so well-established that it no longer requires emphasis, it is perhaps worth pointing out that in the most recent UK national audit of lung cancer practice in 2008 , only $76 \%$ of patients in the UK, England and Wales, had a CT prior to FB [29]. 
In most UK centres bronchoscopy is performed, in the majority of cases, by the chest physicians, with many regarding it as an essential part of their activities [22]. This study supports the establishment of a specialist, multidisciplinary bronchoscopy team, and of cancer multidisciplinary team working in general. The concentration of cases suitable for TBNA on the bronchoscopy list of the lead clinician for lung cancer and bronchoscopy permitted the development of expertise in TBNA without diluting the experience across several operators. Collaboration between chest physicians and pathologists, and the appointment of a specialised bronchoscopy nurse, facilitates optimal preparation of cytological specimens, but these measures are not easily amenable to quantification. The influence of the pathologist on the likelihood of a positive bronchoscopic diagnosis is important. It is easier for a pathologist to give a confident positive malignant diagnosis if he or she has access to full clinical and radiological data via a chest multi-disciplinary team meeting, particularly for cytological samples with scant cellular material or where a bronchial biopsy is negative but the cytology appears malignant. The highly significant influence of year of study in multiple regression analysis of our data is a surrogate for the measured and unmeasured variables that changed over the course of our study. As such we believe it may represent the impact of developing a specialist, collaborative team devoted to optimum lung cancer care.

During our study the numbers of FBs performed for lung malignancy fell. This may have been due to increasing use of less invasive techniques, such as ultrasound-guided fineneedle aspiration of supraclavicular lymph nodes [30], and better case selection. It is a weakness of our study that we did not collect data on the proportion of all lung cancer patients diagnosed bronchoscopically, or on the average number of diagnostic procedures per patient per year. It would be expected, within a high-quality lung cancer service, that the latter number would be minimised. These would be appropriate subjects for further study.

\section{Conclusion}

This study suggests that simple, inexpensive and readily implemented service developments may significantly improve the detection rate of FB for lung malignancy. In particular, it is recommended that all lung cancer diagnostic bronchoscopy should be performed by teams who have access to and develop expertise in TBNA. Bronchial brushes having a larger diameter appear to offer a higher diagnostic yield. Future BTS guidelines on bronchoscopy should set higher targets for the detection rate for lung cancer.

\section{SUPPORT STATEMENT}

F.V. Gleeson is supported by the NIHR biomedical research centre in Oxford (UK).

\section{STATEMENT OF INTEREST}

None declared.

\section{REFERENCES}

1 Cancer Research UK. Lung cancer and smoking statistics - key facts. http://info.cancerresearchuk.org/cancerstats/types/lung/ Date last updated: July 12, 2010. Date last accessed: May 1, 2010.
2 Rivera MP, Mehta AC, American College of Chest Physicians. Initial diagnosis of lung cancer: ACCP evidence-based clinical practice guidelines (2nd edition). Chest 2007; 132: Suppl. 3, 131S-148S.

3 Laroche C, Fairbairn I, Moss H, et al. Role of computed tomographic scanning of the thorax prior to bronchoscopy in the investigation of suspected lung cancer. Thorax 2000; 55: 359-363.

4 National Collaborating Centre for Acute Care. The Diagnosis and Treatment of Lung Cancer: Methods, Evidence and Guidance. London, National Institute for Clinical Excellence, 2005. www. nice.org.uk/nicemedia/pdf/cg024fullguideline.pdf.

5 Schenk DA, Bryan CL, Bower JH, et al. Transbronchial needle aspiration in the diagnosis of bronchogenic carcinoma. Chest 1987; 92: 83-85.

6 Shah PL, Singh S, Bower M, et al. The role of transbronchial fine needle aspiration in an integrated care pathway for the assessment of patients with suspected lung cancer. J ThoracOncol 2006; 1: 324-327.

7 Herth F, Becker HD, Ernst A. Conventional vs endobronchial ultrasound-guided transbronchial needle aspiration: a randomized trial. Chest 2004; 125: 322-325.

8 Herth FJ, Eberhardt R, Vilmann P, et al. Real-time endobronchial ultrasound guided transbronchial needle aspiration for sampling mediastinal lymph nodes. Thorax 2006; 61: 795-798.

9 Gildea TR, Mazzone PJ, Karnak D, et al. Electromagnetic navigation diagnostic bronchoscopy: a prospective study. Am J Respir Crit Care Med 2006; 174: 982-989.

10 Schwarz Y, Mehta AC, Ernst A, et al. Electromagnetic navigation during flexible bronchoscopy. Respiration 2003; 70: 516-522.

11 The Royal College of Pathologists. Histopathology and Cytopathology of Limited or No Clinical Value. 2nd Edn. London, Royal College of Pathologists, 2005. www.rcpath.org/ resources/pdf/holncv-2ndedition.pdf.

12 Lee $\mathrm{CH}$, Wang $\mathrm{CH}$, Lin $\mathrm{MC}$, et al. Multiple brushings with immediate Riu's stain via flexible fibreoptic bronchoscopy without fluoroscopic guidance in the diagnosis of peripheral pulmonary tumours. Thorax 1995; 50: 18-21.

13 Buccheri G, Barberis P, Delfino MS. Diagnostic, morphologic, and histopathologic correlates in bronchogenic carcinoma. A review of 1,045 bronchoscopic examinations. Chest 1991; 99: 809-814.

14 McLean AN, Douglas JG, Semple PD. Scottish national bronchoscopy audit: a prospective multicentre study of 3316 cases against agreed standards. Scottish Thoracic Society. Respir Med 2000; 94: 511-515.

15 McLean AN, Semple PA, Franklin DH, et al. The Scottish multicentre prospective study of bronchoscopy for bronchial carcinoma and suggested audit standards. RespirMed 1998; 92: 1110-1115.

16 British Thoracic Society Bronchoscopy Guidelines Committee, a Subcommittee of the Standards of Care Committee of the Brithish Thoracic Society. British Thoracic Society guidelines on diagnostic flexible bronchoscopy. Thorax 2001; 56: Suppl. 1, i1-i21.

17 Haponik EF, Cappellari JO, Chin R, et al. Education and experience improve transbronchial needle aspiration performance. Am J Respir Crit Care Med 1995; 151: 1998-2002.

18 Hsu LH, Liu CC, Ko JS. Education and experience improve the performance of transbronchial needle aspiration: a learning curve at a cancer center. Chest 2004; 125: 532-540.

19 Hermens FH, Limonard GJ, Termeer R, et al. Learning curve of conventional transbronchial needle aspiration in pulmonologists experienced in bronchoscopy. Respiration 2008; 75: 189-192.

20 Wang KP, Terry PB. Transbronchial needle aspiration in the diagnosis and staging of bronchogenic carcinoma. Am Rev Respir Dis 1983; 127: 344-347.

21 Haponik EF, Russell GB, Beamis JF Jr, et al. Bronchoscopy training: current fellows' experiences and some concerns for the future. Chest 2000; 118: 625-630. 
22 Smyth CM, Stead RJ. Survey of flexible fibreoptic bronchoscopy in the United Kingdom. Eur Respir J 2002; 19: 458-463.

23 Harrow EM, Abi-Saleh W, Blum J, et al. The utility of transbronchial needle aspiration in the staging of bronchogenic carcinoma. Am J Respir Crit Care Med 2000; 161: 601-607.

24 Holty JE, Kuschner WG, Gould MK. Accuracy of transbronchial needle aspiration for mediastinal staging of non-small cell lung cancer: a meta-analysis. Thorax 2005; 60: 949-955.

25 Lundgren R, Bergman F, Angstrom T. Comparison of transbronchial fine needle aspiration biopsy, aspiration of bronchial secretion, bronchial washing, brush biopsy and forceps biopsy in the diagnosis of lung cancer. Eur J Respir Dis 1983; 64: 378-385.

26 Gasparini S, Zuccatosta L, Zitti P, et al. Integration of TBNA and TCNA in the diagnosis of peripheral lung nodules. Influence on staging. Ann Ital Chir 1999; 70: 851-855.
27 Bilaceroglu S, Gunel O, Cagirici U, et al. Comparison of endobronchial needle aspiration with forceps and brush biopsies in the diagnosis of endobronchial lung cancer. Monaldi Arch Chest Dis 1997; 52: 13-17.

28 Gaeta M, Pandolfo I, Volta S, et al. Bronchus sign on CT in peripheral carcinoma of the lung: value in predicting results of transbronchial biopsy. AJR Am J Roentgenol 1991; 157: 1181-1185.

29 NHS Information Centre. National Lung Cancer Audit 2009. London, NHS Information Centre, 2009. www.ic.nhs.uk/webfiles/ Services/NCASP/audits\%20and\%20reports/NHS\%20IC\%20Lung \%20Cancer\%20AUDIT\%202009\%20FINAL.pdf.

30 Kumaran M, Benamore RE, Vaidhyanath R, et al. Ultrasound guided cytological aspiration of supraclavicular lymph nodes in patients with suspected lung cancer. Thorax 2005; 60: 229-233. 